\title{
Article \\ Geo-Electrical Detection of Impermeable Membranes in the Subsurface
}

\author{
Marios Karaoulis ${ }^{1, *}$, Pauline P. Kruiver ${ }^{2}\left(\mathbb{D}\right.$, Victor Hopman ${ }^{1}$ and Bob Beuving ${ }^{3}$ \\ 1 Deltares, Subsurface \& Groundwater Systems, 3508 AL Utrecht, The Netherlands; victor.Hopman@deltares.nl \\ 2 Royal Netherlands Meteorological Institute, 3730 AE De Bilt, The Netherlands; pauline.kruiver@knmi.nl \\ 3 Beuving Civiel Advies \& Management, 1213 AG Hilversum, The Netherlands; bob@beuvingciviel.nl \\ * Correspondence: marios.karaoulis@deltares.nl
}

check for

updates

Citation: Karaoulis, M.; Kruiver, P.P.;

Hopman, V.; Beuving, B.

Geo-Electrical Detection of

Impermeable Membranes in the

Subsurface. Appl. Sci. 2022, 12, 1555.

https://doi.org/10.3390/

app12031555

Academic Editors: Yangquan Chen, Simone Morais, Subhas

Mukhopadhyay, Junseop Lee, M.

Jamal Deen and Nunzio Cennamo

Received: 3 January 2022

Accepted: 28 January 2022

Published: 31 January 2022

Publisher's Note: MDPI stays neutral with regard to jurisdictional claims in published maps and institutional affiliations.

Copyright: (c) 2022 by the authors Licensee MDPI, Basel, Switzerland. This article is an open access article distributed under the terms and conditions of the Creative Commons Attribution (CC BY) license (https:// creativecommons.org/licenses/by/ $4.0 /)$.
Featured Application: Looking ahead capabilities of electrical resistivity tomography (ERT) for a soil penetrating tool.

\begin{abstract}
In this paper, we have investigate looking ahead capabilities of electrical resistivity tomography (ERT) for a soil penetrating tool with electrodes. In our case study, the desired detection resolution (10 to $20 \mathrm{~cm}$ at a depth of at least $6 \mathrm{~m}$ ) was much higher than what can be achieved from classical surface ERT measurements. Therefore, we designed a logging-type tool, that can be pushed into the ground. Our target was a buried PVC membrane which acts as an electrical insulator. In this phase, we performed numerical simulations and laboratory measurements. The methodology is based on a two-step approach. First, we calculate the background resistivity of the tool's path is determined by inversion of near-looking electrode configurations. Next, the theoretical response (kernel) of the far-looking configurations is calculated for different membrane positions. The root mean square (RMS) error between the kernel and the measurements is minimized to detect the membrane. If the membrane is within sensing reach, the RMS has a minimum for the kernel corresponding to the true membrane position. If no minimum in RMS is found, the membrane is not within sensing reach and the tool can be pushed closer to the membrane. The laboratory tests comprised measurements in a tank filled with either water or saturated sand or saturated sand with clay slabs and chunks. The laboratory results were successful in pinpointing the position of the membrane with an accuracy of 10 to $20 \mathrm{~cm}$, depending on the dimension of the tool and the distance from the membrane.
\end{abstract}

Keywords: look-ahead; detection of impermeable membranes; electrical resistivity tomography; geophysics

\section{Introduction}

Infrastructure works in low-lying countries with high water tables occasionally require measures to prevent groundwater from flooding the structure. An example is the construction of a road below the groundwater table. In the case at hand, the road has been constructed in a large trench which is lined with an impermeable membrane in the 1980's. Due to the increased traffic load, a lane needs to be added to the road inside the trench. The road is of vital importance for the region and cannot be closed during the reconstruction and excavation works. For safety and to avoid flooding during excavation and construction, it is important to know the exact position of the membrane. The goal was to locate the membrane with an accuracy of 10 to $20 \mathrm{~cm}$ at a depth of 6 to $10 \mathrm{~m}$. The approximate location of the membrane is available from archive design drawings. The actual location needs to be established through measurements. Geophysical methods that operate from the surface do not have sufficient accuracy. Therefore, the measurement tool needs to be inserted into the soil enabling measurements to be taken from a closer distance to the membrane. The difficulty is that the PVC membrane has a thickness of less than $1 \mathrm{~mm}$. In addition, the tool needs to be kept at a "safe" distance of approximately $1 \mathrm{~m}$ from the 
theoretical (design) position of the membrane. Puncturing the membrane is to be avoided at all costs, because flooding of the road would result in serious economic damage.

A relevant physical contrast that is present between the membrane and the surrounding soil is the electrical resistivity. Electrical Resistivity Tomography (ERT) is a geophysical method commonly used in many near-surface applications (e.g., [1]) and has a large range of resistivity values that can be imaged, from very conductive to very resistive. In our case, the membrane acts as an insulator and has an electrical resistivity in the order of $10^{10} \mathrm{Ohm} \cdot \mathrm{m}$. The partially saturated sand on top of the membrane has electrical resistivity values varying from 15 to $800 \mathrm{Ohm} \cdot \mathrm{m}$. Because of this large difference, the electrical resistivity tomography method was selected and adjusted for the present application.

In this research, we explore the detection and looking-ahead capabilities of a custommade tool that penetrates the soil. Because of the membrane's function as a liner against groundwater leakage, it should stay intact during reconstruction works. It is therefore important for our method to be able to "look ahead" and notice that the membrane is getting close. Measuring ERT from the surface results in a depth resolution that is too poor for the application. Measuring from boreholes is a definite no-go in this case, because the boreholes might penetrate the membrane. We therefore developed a tool that can penetrate the ground and look ahead to check whether it is safe to penetrate the tool some further distance and measure again, until membrane is located with the desired resolution.

\section{Principles of ERT}

Electrical resistivity tomography (ERT) is a geophysical technique that has traditionally been used for imaging sub-surface structures. It can be applied using electrodes from the surface or using electrodes in one or more boreholes. The electrical currents in the subsurface are influenced by the composition of the matrix (e.g., sand, gravel, clay) and the composition of the water in the pores. In principle, the resistivity is measured using electrodes at different positions. A tomographic image of the resistivity distribution of the subsurface is obtained through inversion.

A typical acquisition system for Direct Current resistivity measurements is shown in Figure 1a,b. The basic setup comprises a four-electrode array with a voltmeter and a current meter. For each measurement, two current electrodes are used: one to inject the current into the subsurface or waterbody and the other to retrieve the same amount of current from it. By convention, these electrodes are named A and B, respectively. The electric field is measured (at least) with two other electrodes ( $\mathrm{M}$ and $\mathrm{N}$ ), called the potential electrodes. The way in which the current and potential electrodes are arranged is called an array. The choice of a particular resistivity array for a survey is based upon considerations regarding theoretical advantages and drawbacks of the array and its signal-to-noise ratio [2]. Some of the arrays utilize remote electrodes, i.e., electrodes that are installed relatively far away and can be considered as infinitely far. In general, as the distance between the electrodes increases, the depth of investigation, or in the volume through which the current flows, also increases.

The main characteristic of an array is its geometrical factor, which is uniquely related to the respective distances between the different current and potential electrodes. The geometrical factor $g$ describes the relation between the so-called apparent resistivity $\rho_{a}$ and the measured resistance $R$ which represents a weighted average of the true resistivity of the subsurface:

$$
\rho_{a}=R \cdot g
$$

The apparent resistivity is equal to the true resistivity in the case of homogeneous material. The equation of the geometrical factor, in case of half space, depends only on the position of the electrodes. For special cases, e.g., a laboratory experiment in a tank, the geometrical factor needs to be numerically calculated. The true resistivity can be obtained after inversion of the apparent resistivity data. The inversion procedure aims to find a resistivity model that explains (or "predicts") the given field measurements best according to a misfit definition. More detailed information about the ERT method can be found in [1]. 

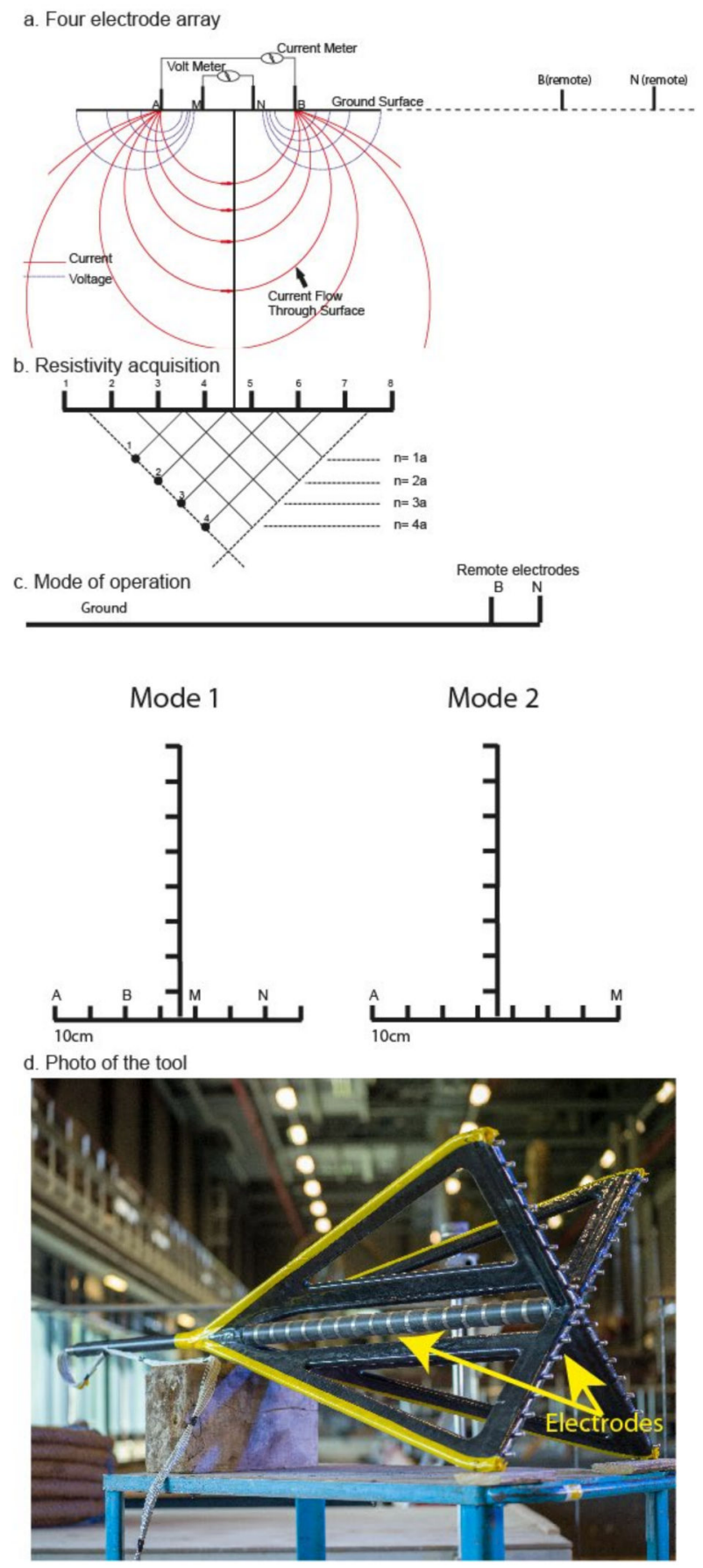

Figure 1. (a) A typical ERT system with the two current electrodes (A and B) and two potential electrodes ( $\mathrm{M}$ and $\mathrm{N}$ ). (b) Schematic representation of an array of electrodes. (c) Cross-section showing the two modes used in the simulation in a T-shaped tool. (d) Prototype of ERT tool with ring electrodes on the pole and pin electrodes on the cross.

The ERT method is used in a variety of applications such as in hydrogeology and for the assessment of geohazards. Examples are imaging of sinkholes [3], cavities [4], landslides [5]. It is also one of the most widely used near-surface geophysical survey for civil engineering applications [6]. Other more recent applications can be found in [1].

In a typical application of the ERT method, the electrodes are placed on the surface and a tomographic image of the subsurface can be obtained. The depth of investigation relates to the electrode spacing, total number of electrodes used and the geoelectrical properties of the subsurface. The spatial resolution of the recovered model is decreasing with the 
increasing depth, making it harder to determining the exact positioning of deeper targets. Electrodes can also be placed inside boreholes to increase the resolution at depth, typically using dipole-dipole and pole-dipole arrays [7]. Alternative to ERT, is the use of logging tools (i.e., Lateral $\log [8,9])$. Those tools though are optimized to measure in the very close vicinity of the logging tool.

For our case of finding the position of the PVC membrane and a safe excavation depth, we performed several classical ERT lines with electrodes on the surface, parallel to the road on the roadside slope. We used a cable with $2.00 \mathrm{~m}$ spacing between the electrodes and a total number of 81 electrodes. The array configurations were gradient and dipole-dipole with a total number of 4617 measurements. The measurements were inverted to resistivity profiles (Figure 2). The overall root-mean-square (RMS) error of the inversions was around $8.5 \%$. This rather high RMS is probably due to the urban environment (highway, artifacts, lampposts), which causes electrical noise. The inversion result (Figure 2) shows a simple resistivity structure of the subsurface, with a zone of $8 \mathrm{~m}$ unsaturated sand, followed by $6 \mathrm{~m}$ of saturated sand with some clay pockets. The membrane is visible at 14 to $16 \mathrm{~m}$ depth, but the resolution of ERT in those depths is about $1 \mathrm{~m}$ (or more) in the vertical scale. This result is insufficient for our case. Still, this investigation was useful for obtaining information about the background resistivity and information about the degree of heterogeneity.

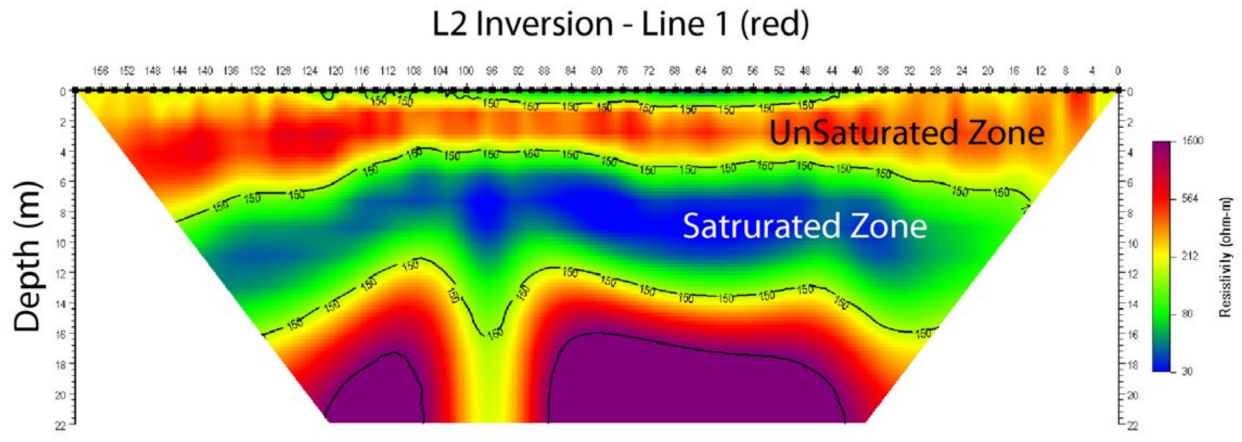

L2 Inversion - Line 2 (green)

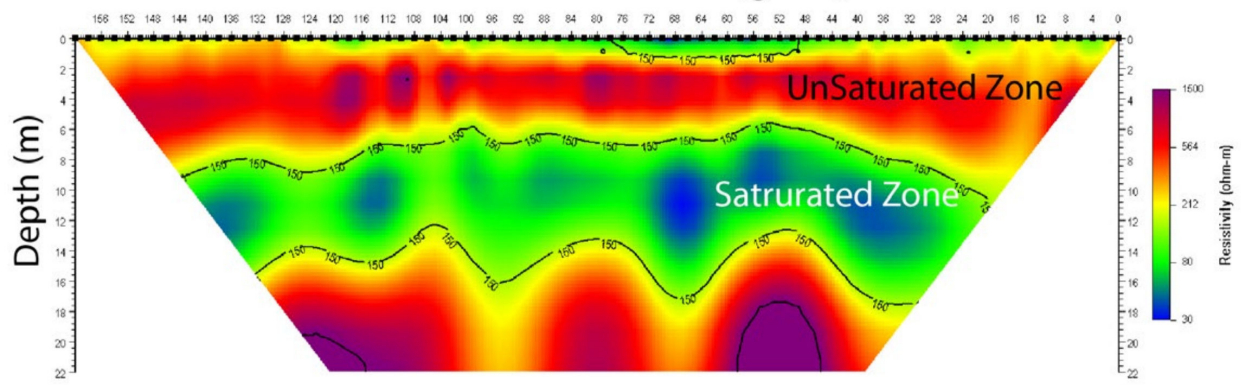

Figure 2. Two surface ERT lines aimed at detecting the membrane from the surface. The lines were situated parallel to the road on the slope of the roadside. The membrane shows as a very high resistive target (more than $1000 \mathrm{Ohm} \cdot \mathrm{m}$ ) at a depth of $14-16 \mathrm{~m}$. The vertical resolution is about $1 \mathrm{~m}$, i.e., not sufficient to pinpoint the positioning with the desired resolution.

In order to increase the resolution, the electrodes need to be closer to the target. We therefore need a tool that penetrates the soil and is able to look ahead to check whether the target (PVC membrane) is near. Our idea of looking ahead is inspired by two similar techniques known as borehole logging and Tunnelling Boring Machines (TBM). During borehole logging, a clay-mud is required providing both stability to the borehole and good electrical contact [10]. The very first logging tools utilized 4 electrodes arrays only. In these cases, current may be confined within the more conductive mud, especially when mud with higher salinity, and therefore missing the desired target. To overcome this problem, new logging tools were developed that utilize the simultaneous use of an array of electrodes, 
achieving focused currents into the formation and avoiding the effect of the mud. Since the initial development of focused arrays for borehole applications, many unique methods for focusing have been manufactured for commercial use (Laterolog LLS, Laterolog LLD, HRLA Dual Laterolog etc., [8,9]). In our case, however, boreholes are not allowed.

Another application with looking ahead capabilities originates from tunnelling. Operators needed predictions of soil conditions ahead of the cutter face. Literature on the details of these methods is limited to the PhD thesis of Schaeffer [11]. There are two commercial systems available, called the Bore-tunneling Electrical Ahead Monitoring (BEAM) system [12] and the BEAM4 [13]. These systems were developed over the last two decades to be fully integrated into TBMs. Both systems use electrical resistivity and induced polarization (IP) methods to characterize the material ahead of the tunnel face. They make clever use of the cutterheads of the TBM as electrodes. The system jumps between two different modes, one that uses the focusing electrodes to image further ahead and one scan mode to look in the vicinity of the TBM. The TBM methods, however, are patent-pending and therefore scientific literature is not available apart from [12,13].

\section{Concept of Looking Ahead}

A simulation exercise was performed as a preparation for the prototype design and the laboratory test. As mentioned, a larger separation of the electrodes allows the current to flow deeper in the subsurface and subsequently to look further ahead. However, since the electrodes will be implemented into a sort of logging tool there are limitations on the dimension of the tool and electrode spacing. Our tool has a " $\mathrm{T}$ " shape with electrodes on the horizontal and vertical axes (Figure 1d). There are also remote electrodes, installed on the surface far away from the tool. Remote electrodes are typically positioned about 10 times the depth of target away. Two modes are defined, which are schematically sketched in Figure 1c:

1. Mode 1: All electrodes are located on the horizontal axis of the tool. No remote electrodes are being used.

2. Mode 2: At least two electrodes are located on the horizontal axis and use of remote electrodes ( 1 or 2 depending on the array).

A variation of these modes would be using electrodes on the vertical axis, or a combination of electrodes from the horizontal and the vertical axes. Modes 1 and 2, however, demonstrate the idea of looking ahead. We expect that Mode 1 is sensitive to the resistivity structure close to the tool, because the two current electrodes are located on the tool. We refer to this configuration as near-looking. In Mode 2, however, the current will travel through a much larger volume, because of the position on the remote electrodes. We expect this mode to be sensitive to the resistivity further away from the tool. We refer to this configuration as far-looking. The final tool will include a combination of all modes and electrodes, as we will explain in the later section.

All simulations are performed using COMSOL (www.comsol.com, accessed on 1 June 2018). We simulate a homogeneous saturated sand body with a resistivity of $30 \mathrm{Ohm} \cdot \mathrm{m}$ and an insulator (i.e., membrane) at $6.00 \mathrm{~m}$ depth. For every location of the tool and for every possible location of the membrane, we simulate the forward response of the measurements, which we call kernel. In other words, the kernel is the theoretical response of tool, if we know the background resistivity structure and the location of the membrane. This kernel will be the basis of the calculations. The kernels were calculated for Mode 1 and Mode 2 for a range of electrode spacings (Figure 3). For example, the light blue line in Figure 3 (left panel) shows the model resistivity result for a spacing between $\mathrm{A}$ and $\mathrm{N}$ of $60 \mathrm{~cm}$. For Mode 2 (Figure 3, right panel), the number in the legend refers to the spacing between the electrodes $\mathrm{A}$ and $\mathrm{M}$, since only two electrodes are used on the tool in this configuration. The vertical axis indicates the position of the tool at various distances relative to the membrane.

The looking ahead capabilities are qualitatively assessed by the position of the curvature of the kernels in Figure 3. We consider the position where the curvature is changing as detection distance. As expected for Mode 1, the array is mostly influenced by the resistivity 
in the vicinity of the four electrodes on the tool. Consequently, the array needs to be relatively close to the membrane to "sense" it. The increased electrode spacing only slightly increases the capability of looking ahead. For example, when the array is $10 \mathrm{~cm}$ wide (corresponding to $3.3 \mathrm{~cm}$ electrode spacing), the membrane can be sensed at $20 \mathrm{~cm}$ distance (at a depth of $580 \mathrm{~cm}$ ). When the array is much bigger, e.g., $60 \mathrm{~cm}$, it senses the membrane at a distance of $60 \mathrm{~cm}$ (depth of $540 \mathrm{~cm}$ ). For Mode 2, however, the presence of the membrane is sensed by the tool at a much larger distance. The curves presented here are shown as part of the explanation of the methodology, in Section 8 we discuss the process of determining the position of the membrane.
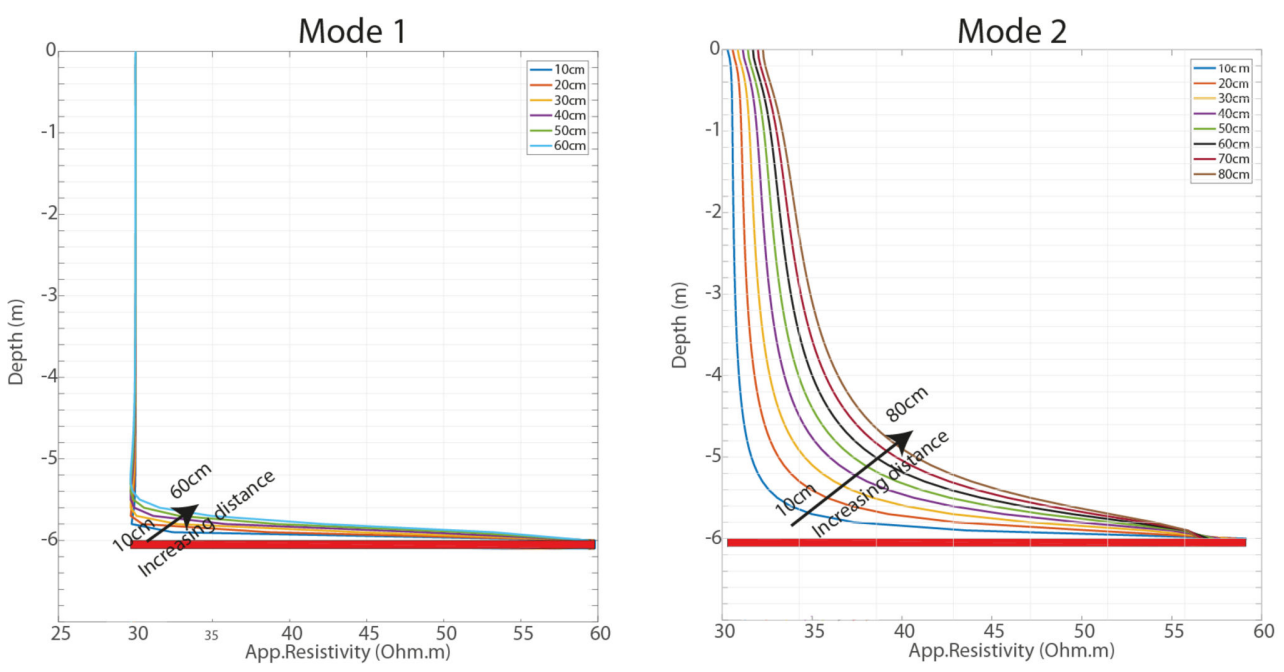

Figure 3. Sensitivity study for two modes (see Figure $1 \mathrm{c}$ for electrode configuration) and their looking-ahead capabilities. The legend shows the largest distance between the electrodes (A and $\mathrm{N}$ for mode 1; $\mathrm{A}$ and $\mathrm{M}$ on the tool for mode 2, $\mathrm{B}$ and $\mathrm{N}$ are remote). The curves represent kernels.

In the field situation, the ground above the membrane might not be homogeneous (as in the simulation) and the background resistivities might be unknown. Therefore, a combination of different modes is required for local resistivity information about the material the tool is passing through and in order to update the kernel for the approach of the membrane. In the next sections, this so-called on-the-fly calculation of this kernel will be explained.

\section{Laboratory Setup}

An experimental setup was created in the laboratory to test the ERT concept array for membrane detection. The setup consisted of a PE tank of $7.00 \mathrm{~m}$ long, $3.50 \mathrm{~m}$ wide and $2.50 \mathrm{~m}$ deep. The PE material ensures that the setup is electrically isolated from the surroundings. A PVC membrane was attached at one of the ends at the inside of the tank. A prototype tool was built, consisting of a pole with ring electrodes and a cross with pin electrodes (Figure 1d). The material between the electrodes was powder-coated for electrical insulation. The distance between the electrodes on the cross and on the pole was $5 \mathrm{~cm}$. The diameter of the cross was $0.80 \mathrm{~m}$. The cross contained 33 electrodes. The length of the pole was $1.00 \mathrm{~m}$ and the pole contained 17 electrodes. In the field, a measuring tool will be pushed vertically into the soil and will approach the membrane while measuring. The long side of the PE tank corresponds to the depth in the field. For practical reasons, such as the height of the roof, the laboratory setup was rotated 90 degrees relative to the field. Also for practical reasons, the tool was pulled away from the membrane in the laboratory, instead of pushed to the membrane as would be the case in the field. In this way, the prototype could be less robust and the concept could be tested. In order to achieve the pulling away from the membrane, the pole of the tool was connected to a crane which pulled the instrument through the tank via two pulleys (Figure 4, right. The tool was supported by a plastic 
rail, because of its weight. Close to the membrane, measurement positions were spaced at 5 or $10 \mathrm{~cm}$ intervals. Further away from the membrane, the measurement interval was increased to 30 to $50 \mathrm{~cm}$. The minimum distance between the membrane and the tool was $0.10 \mathrm{~m}$, the maximum distance was $4.50 \mathrm{~m}$. The remote electrodes consisted of four metal plates and they were placed in the four corners of the tank, on the right boundary $($ at $+7.00 \mathrm{~m})$. The measuring unit is an MPT DAS-1. The numerical simulation for the lab experiment is included in Section 5.
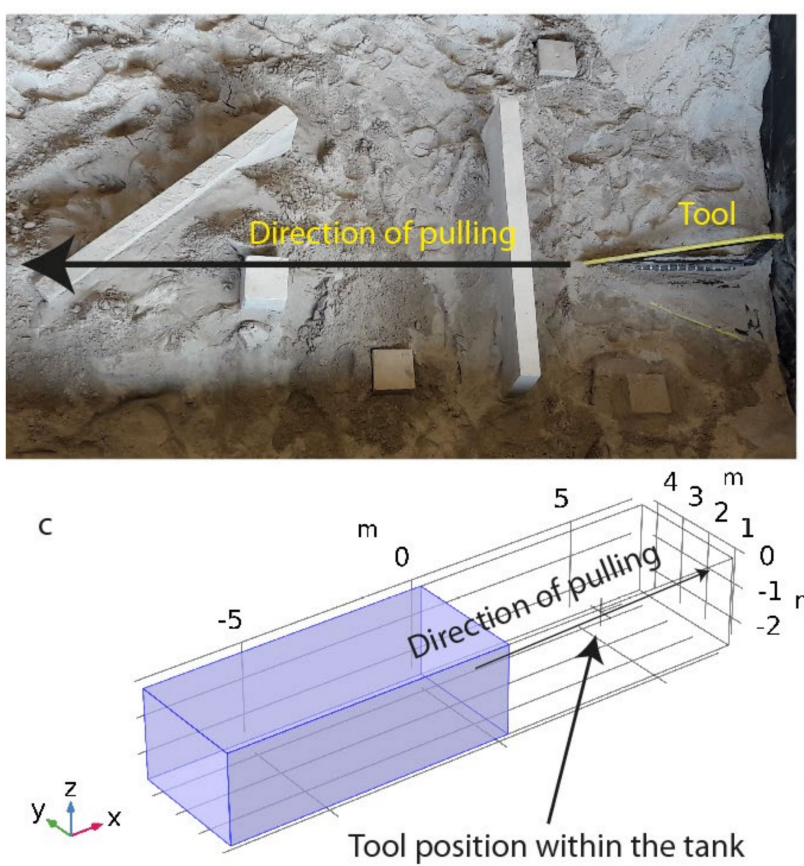

b

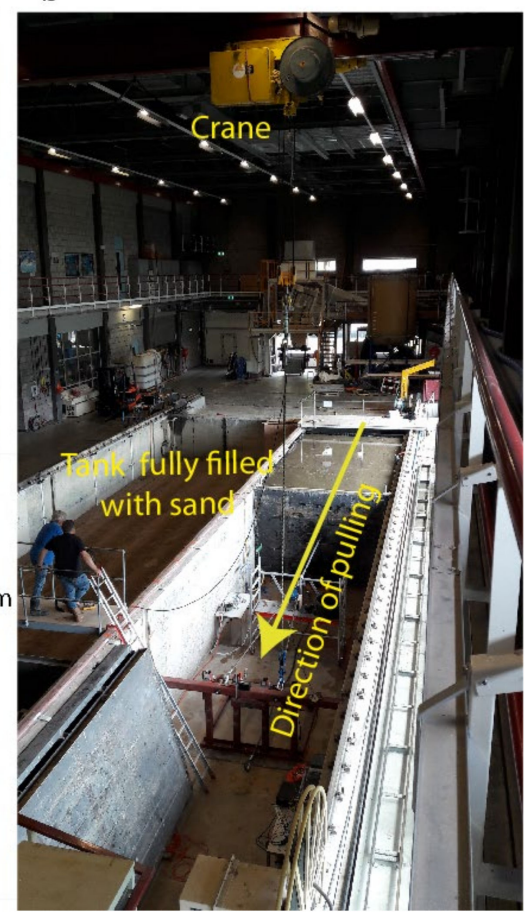

Figure 4. (a) Top view of the prototype of ERT tool with ring electrodes on the pole and pin electrodes on the cross. After filling the tank half full with sand, the clay slabs and cubes and the tool were installed. After that, the tank was filled up with sand and saturated with water. (b) photo of the laboratory setup with the tank, crane and pulleys. (c) Simulation of the tool and tank using COMSOL. The $\mathrm{Y}$ and $\mathrm{Z}$ coordinates are the same as the lab experiment. The actual tank is the white box, where the membrane is located at $0.00 \mathrm{~m}$ along the $\mathrm{YZ}$ plane. The simulation tank is about twice as large in the $X$ dimension as in reality and it spans the white and the blue box. This larger simulation size is required for the calculation of the geometrical factor and the kernels.

In the first test, the tank was filled with tap water creating a homogeneous medium. The tool could move between positions with little effort, because of the absence of friction. Several tests were performed confirming that the tool was working properly. Results from the water filled tank are included in Section 7. For the second test, several slabs and cubes of clay were added to the tank filled with sand (Figure 4a) to study the effect of heterogeneity on the performance of the method. This setup reflects true field conditions with a heterogeneous subsurface to the closest. In general, the soil at the field site consists of sand, but cone penetration tests on the field site have shown that occasionally clay pockets are present. The steps for the installation of the final sand and clay tank were the following:

(1) Install the non-conductive supporting rail

(2) Fill the tank with sand to about half the height (approximately $1 \mathrm{~m}$ ), Figure $4 \mathrm{a}$.

(3) Install the clay slabs and cubes (Figure 4a)

(4) Install the tool (Figure 4a)

(5) Fill the rest of the tank with sand to a height of $2.0 \mathrm{~m}$, to cover the tool and the clay slabs and cubes (Figure 4b) 
(6) Saturate the tank with water

(7) Perform measurements, pull the tool to a new position, repeat measurements, pull the tool etc.

Because of the setup and preparation, the tool was pulled through the tank only once during the tank filled with the sand and clay tank. The results from the experiments are included in Section 5.

\section{Numerical Simulation of the Lab Experiment}

The data collected from the laboratory experiment were used as validation of the methodology. In a laboratory environment, we cannot create a half space with an electrical insulator at depth. The tank has finite dimensions and each side acts is an electrical insulator. The boundary effects are taken into account in the simulations using COMSOL. The actual geometrical factor is calculated numerically from the simulation. This extra step will not be needed in the field situation, because of the true half-space in the field. In the laboratory case, we must calculate the geometrical factor for our specific situation, where each of the six sides of the tank is an insulator.

The model dimensions are shown in Figure 4c. The right part, depicted by the white box, has the dimensions of the actual tank. In order to simulate the response of a membrane at a certain location, we consider five sides of the tank and the position of the tool at each measurement location as known. The only unknown is the 6th side of the tank where the membrane is located (in Figure 4c). It is this side that is our target. In other words, we want to simulate the contribution of the membrane when its position is unknown, within the known dimensions of the tank.

Since the numerical modelling requires a finite space, we increased the modelled length to be about twice the length of the actual one, resulting size of $15.00 \mathrm{~m}$ for the simulation tank. In Figure 4, the simulation tank spans the white and the blue box. The $\mathrm{X}$-axis extends from $-8.00 \mathrm{~m}$ to $+7.00 \mathrm{~m}$. The actual position of the membrane is located at $\mathrm{X}$-location of $0.00 \mathrm{~m}$ in the $\mathrm{YZ}$ plane.

The flow chart of steps for the determination of the position of the membrane is given in Figure 5. The numerical simulation is used to calculate the geometrical factor, the number that multiplied by the measurements in a homogeneous environment (i.e., a water-filled tank) will result in that homogeneous value. The tool measures resistance in Ohm. The geometrical factor is calculated for the simulation tank for all positions of the tool (and thus all electrode locations), starting from $+7.00 \mathrm{~m}$ until $0.00 \mathrm{~m}$. The different positions of the tool represent different distances between the tool and the membrane.

In absence of the membrane the geometrical factor multiplied by the data (synthetic and laboratory) should always result in $21 \mathrm{Ohm} \cdot \mathrm{m}$, which is the resistivity value for the homogenous medium in our case. The next step is the calculation of the kernels. We use the geometrical factor from the simulation tank and the simulated resistance (in Ohm) from the actual tank and simulate various theoretical positions of a membrane. Thus, the increase of resistivity observed when the tool approaches the boundary is due the presence of the left boundary of the 6th side on the actual tank. If the measured data match the kernel for a simulated position of the membrane, then the position of the membrane has been found. If the geometrical factor was calculated based on the actual tank, instead of the simulated tank, we would end up obtaining $21 \mathrm{Ohm} \cdot \mathrm{m}$ for all apparent resistivities. Next, the same geometrical factor is applied to the laboratory data. The kernel is calculated for many theoretical positions of the membrane. If the kernel for a given position and the measured data overlap, then the position of the membrane has been found. The difference or correspondence between a kernel and the laboratory data can be defined by misfit function. In this case, we use the root-mean-square error (RMS) as a metrics for the goodness-of-fit. In preliminary analyses we also tested the area between the curves and the Fréchet [14] as goodness-of-fit metrics. The RMS performed best and we therefore continued using RMS. 


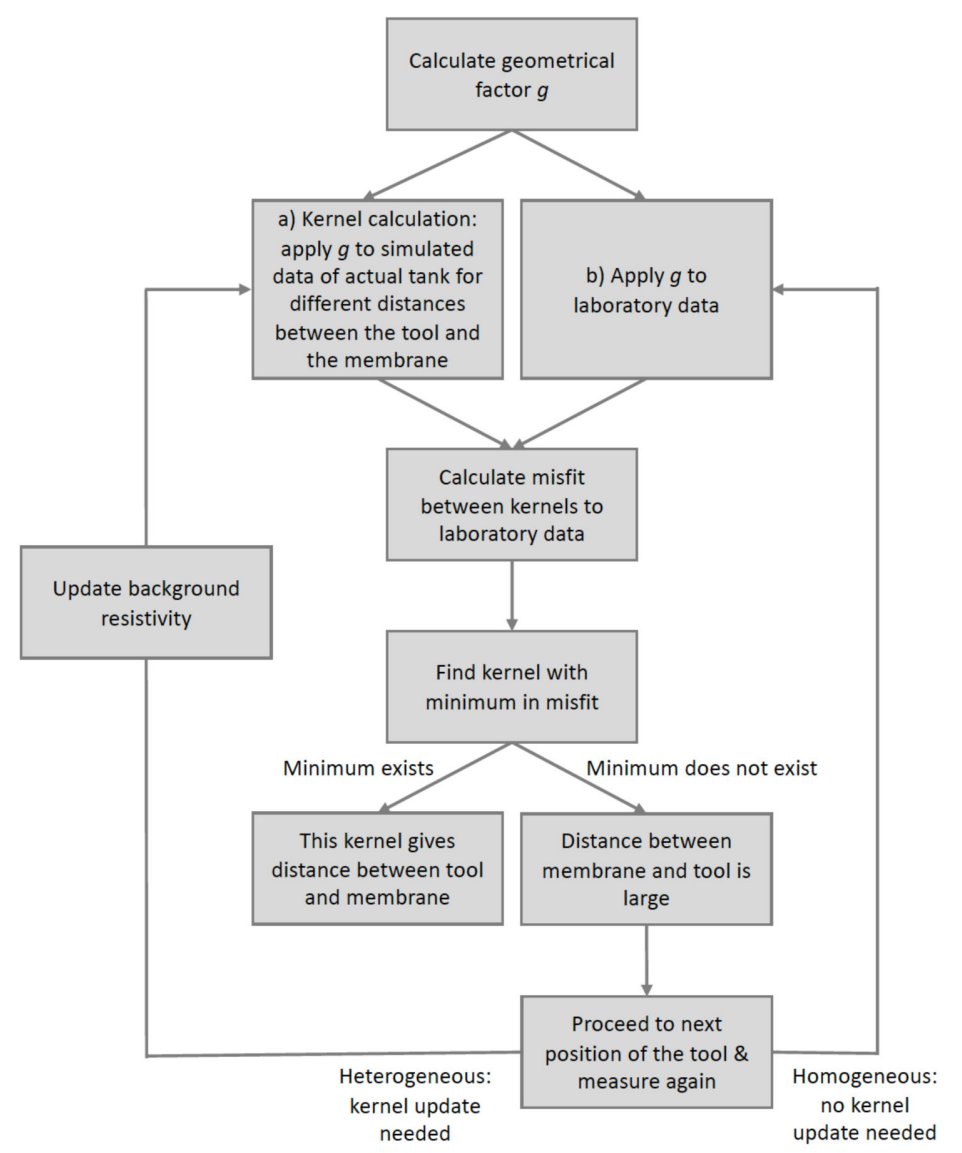

Figure 5. Flowchart of steps for the determination of the position of the membrane. The procedure is repeated several times, until the decision has been made that the tool is close to the tool and its position can be established.

Before each movement of the tool closer to the membrane, all new measurements need to be included in the new kernels. The fact that the kernels need to be updated with new measurements is called "on-the-fly". This is because the processing is done while the tool is in the ground and measurements are being taken and not by processing all data afterwards in the office as is done in a classical ERT survey from the surface (Section 2, Figure 2).

\subsection{Results for Water}

The measured data were compared to the simulated data as a proof-of-concept for the least complicated case, i.e., a water-filled tank. Only the right part of the flow-chart in Figure 5 is used in this example. For the homogenous case, the kernels need to be calculated only once. Figure 6 shows the results from the measured data at discrete positions of the tool (red dots) and simulated data (kernel, blue line), using the two modes and various electrode spacings. Only one kernel is shown, which is the one for the actual position of the membrane. The measured data and simulated data show a good fit. Small variations in measured data originate from the uncertainties in the laboratory, e.g., from small errors in the actual positions of the tool during the experiment, uncertainties in the dimensions of the tool, the finite size of the electrodes and noise in the data. The data using various arrays, among which Modes 1 and 2, were compiled into one dataset for processing. For simplicity, in this paper we present only the data using electrodes along the $\mathrm{Y}$ axis. As expected, the measurements and kernel deviate from the straight line at a larger distance from the membrane for Mode 2 compared to Mode 1 . For both modes, the larger electrode spacing (i.e., a larger tool) results in sensing the membrane from a larger distance. The looking-ahead capabilities of Mode 2 are further increased when using one remote electrode instead of two. Because of the good fit between the simulated data and the laboratory 
results, the simulated data can be used reliably as kernel. The experiment was repeated with a tank filled with saturated sand. The results were comparable to the water-filled tank and therefore not shown.
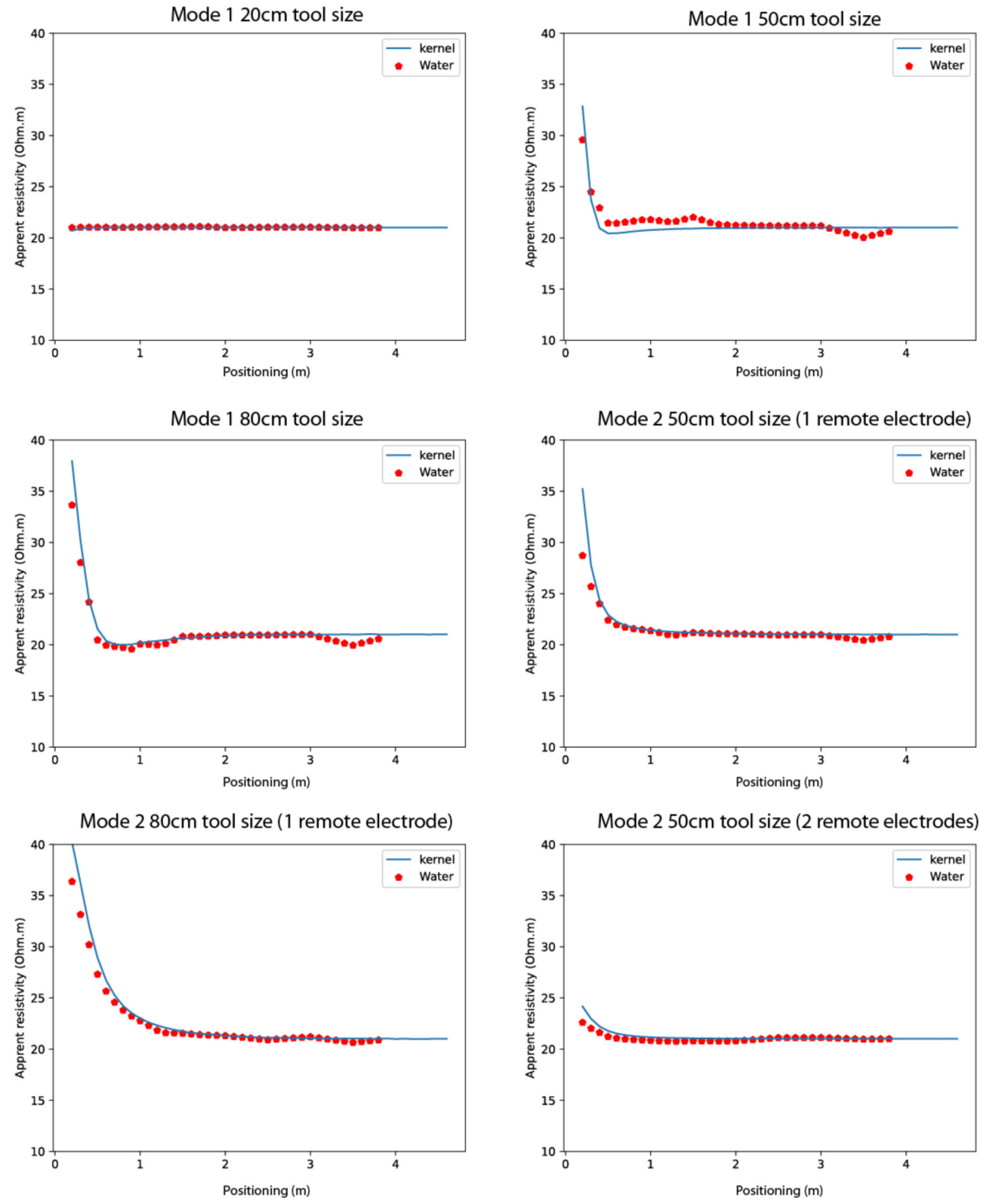

Figure 6. Examples of laboratory data for the water tank (red dots) and kernels calculated for the actual position of the membrane (blue line). Left: For Mode 1 (near-looking) with increasing tool size from top to bottom. Right: For mode 2 (far-looking) with two sizes of a configuration with one remote electrode and one corresponding size with two remote electrodes. The $x$-axis corresponds to the position of the tool, with the membrane located at $x=0 \mathrm{~m}$.

\subsection{Results for Sand and Clay}

The calculation of the kernel requires knowledge of the background resistivity. In the simple homogenous case of the water tank, the kernel could be calculated using the resistivity of the water. In general, however, the background resistivity of the soil is not known. The kernel needs to be built up while more measurement data become available. The "on-the-fly" calculation of the kernel in a heterogeneous environment is described in this section. For this experiment, the same laboratory setup as the water tank was used, but this time clay slabs and cubes of various sizes and shapes were embedded in a tank 
filled with sand (Figure 4, top right and Figure 7, left panels). In this case, the detection of the membrane follows a two-step approach for the box "Kernel calculation" in Figure 5. Step 1 consists of calculating an updated background resistivity for current position of the tool. Step 2 consists of calculating kernels (i.e., membrane positions) at various distances from the current position of the tool. Next, the flow-chart of Figure 5 is followed. The difference between the kernels and the measured data is minimized to determine the most probable distance between the tool and the membrane. The building up of the background resistivity while the experiment progresses (and the tool approaches the membrane) is shown in Figure 7. The steps which are taken to determine whether it is safe to move the tool closer to the membrane or to determine the membrane's position are illustrated with an example in Figures 8-10.
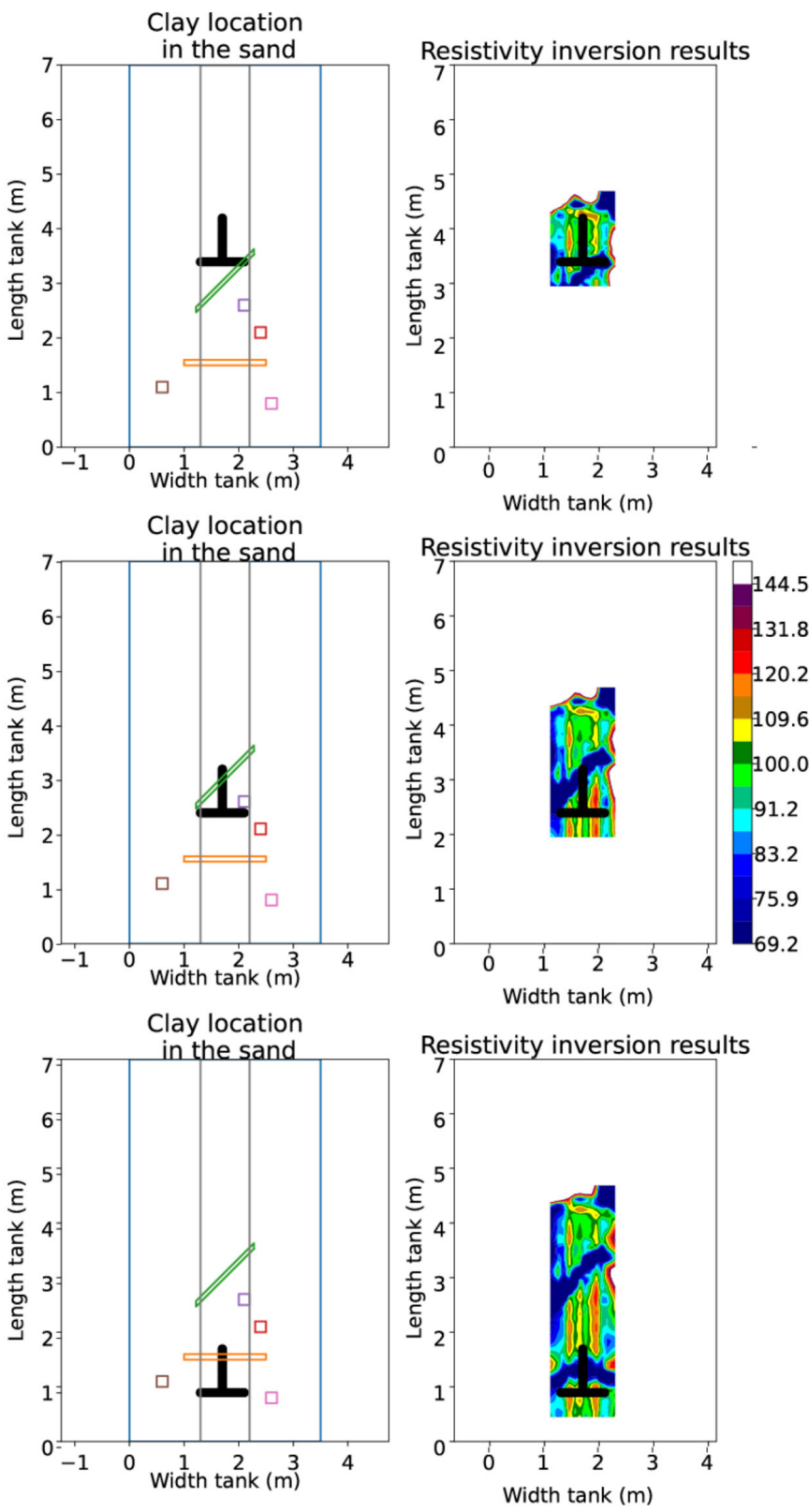

Figure 7. Calculating background resistivity on-the-fly, to produce a new kernel. From top to bottom the tool approaches the membrane which is located at the bottom $(y=0)$. Left: schematic locations of clay slabs and cubes and the position of the tool. Right: background resistivity. A movie of the approach of the tool and the built-up of background resistivity information can be found in the https:/ / youtu.be/N6v_zZTI9pM (accessed on 1 January 2022). 

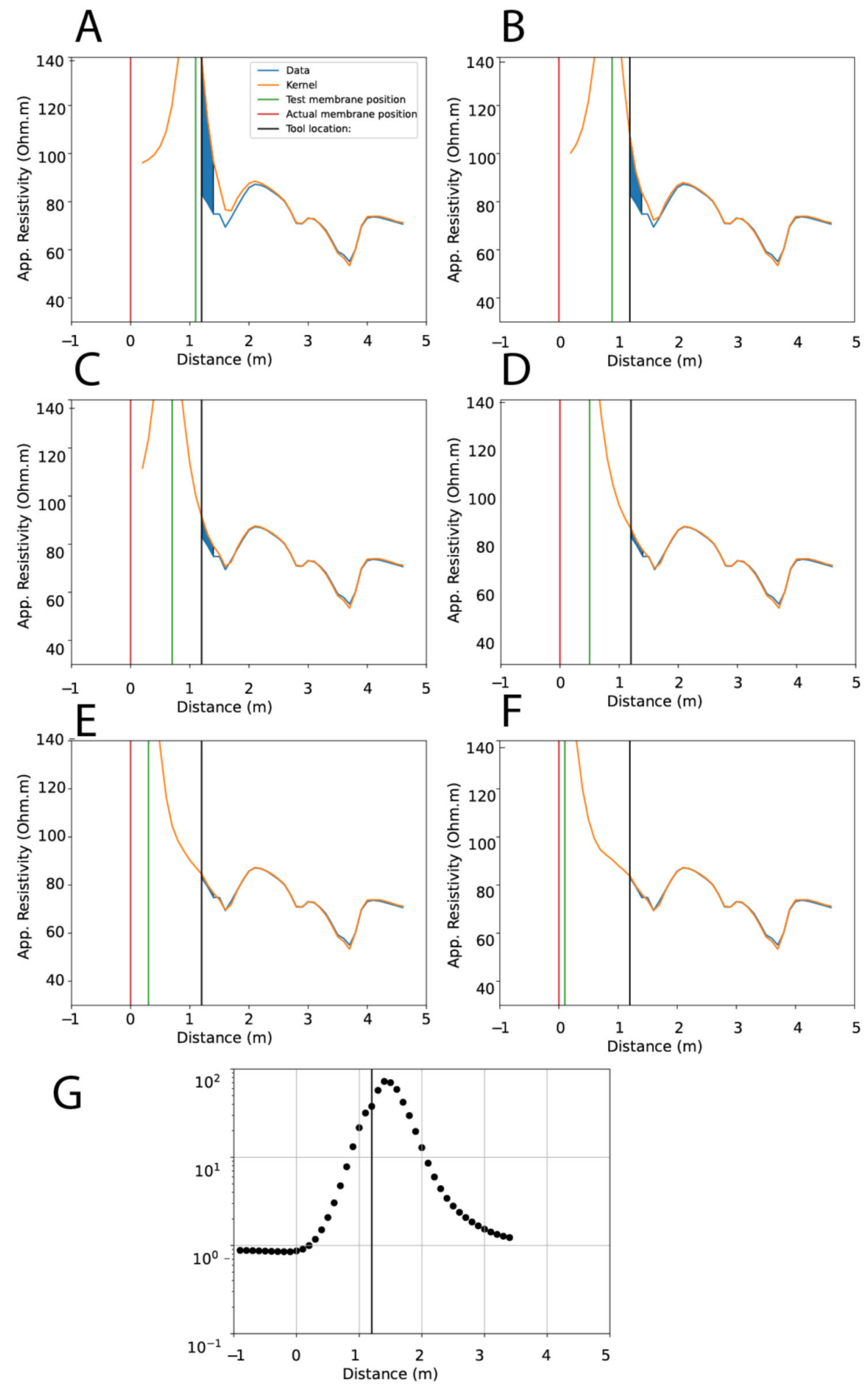

Figure 8. Tool at a fixed position of $+1.2 \mathrm{~m}$ distance from the membrane, represented by the black vertical line. The blue line represents the data acquired up until that position with a Mode 2 array of size $80 \mathrm{~cm}$ (with 1 remote electrode). (A-F) reflect tests for various simulated positions of the membrane. Each panel represents a simulated position of the membrane (green vertical line) with the corresponding kernel shown as the orange line. The red vertical line indicates the true location of the membrane at $x=0.0$. The blue area visualises the misfit between the data (blue line) and kernel (orange line). (G) shows the misfit calculated as RMS between the measured data and the kernel with the membrane at the location of the value on the $x$-axis. In this case, there is no clear global minimal at $0.0 \mathrm{~m}$. This means that the membrane is still far away from the tool and it is safe to push the tool deeper to the next measurement position. 

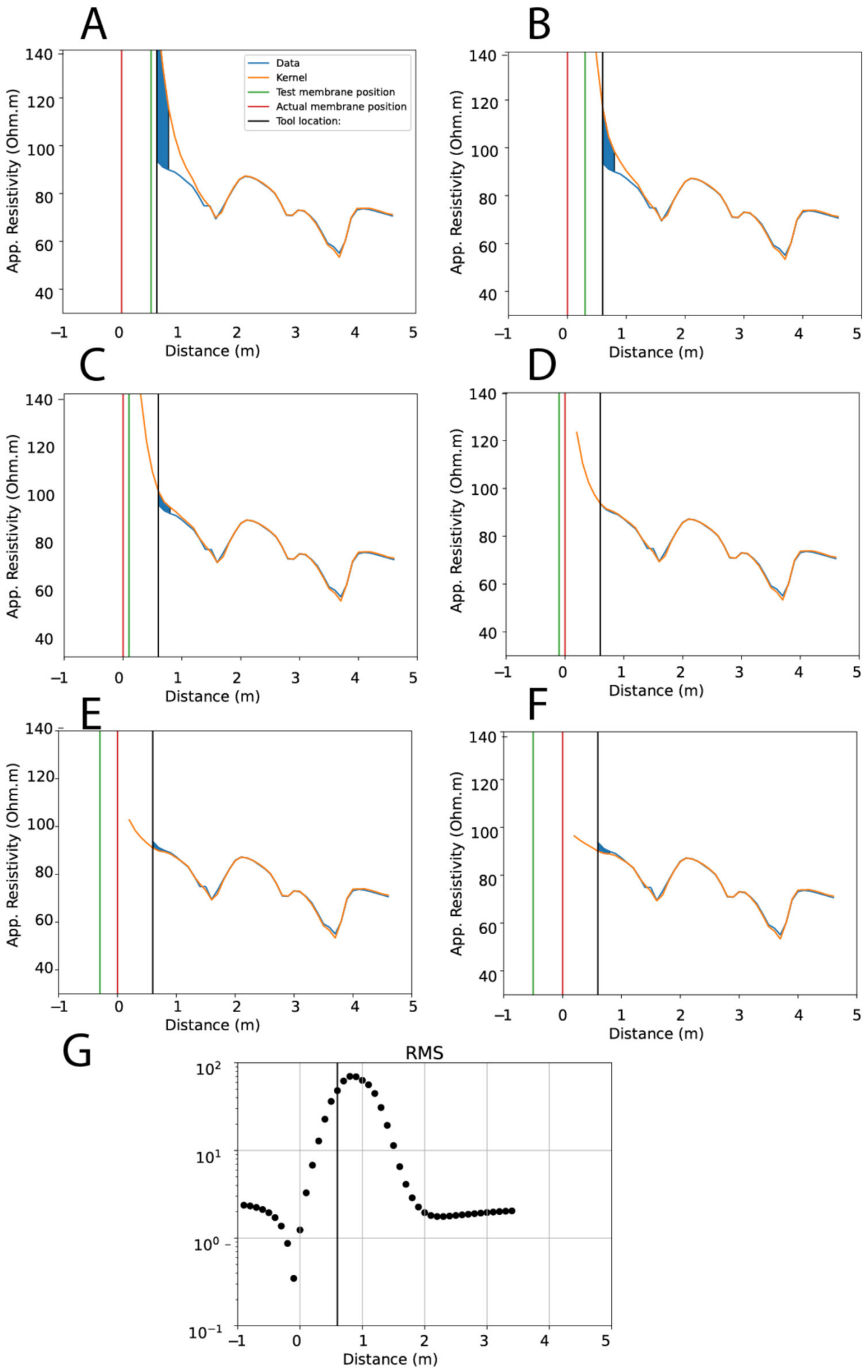

Figure 9. Same as Figure 8 with the tool (black vertical line) at $0.6 \mathrm{~m}$ from the membrane (red vertical line). (A-F) reflect tests for various simulated positions of the membrane. (G) shows the misfit function with a global minimum at $-0.1 \mathrm{~m}$. 

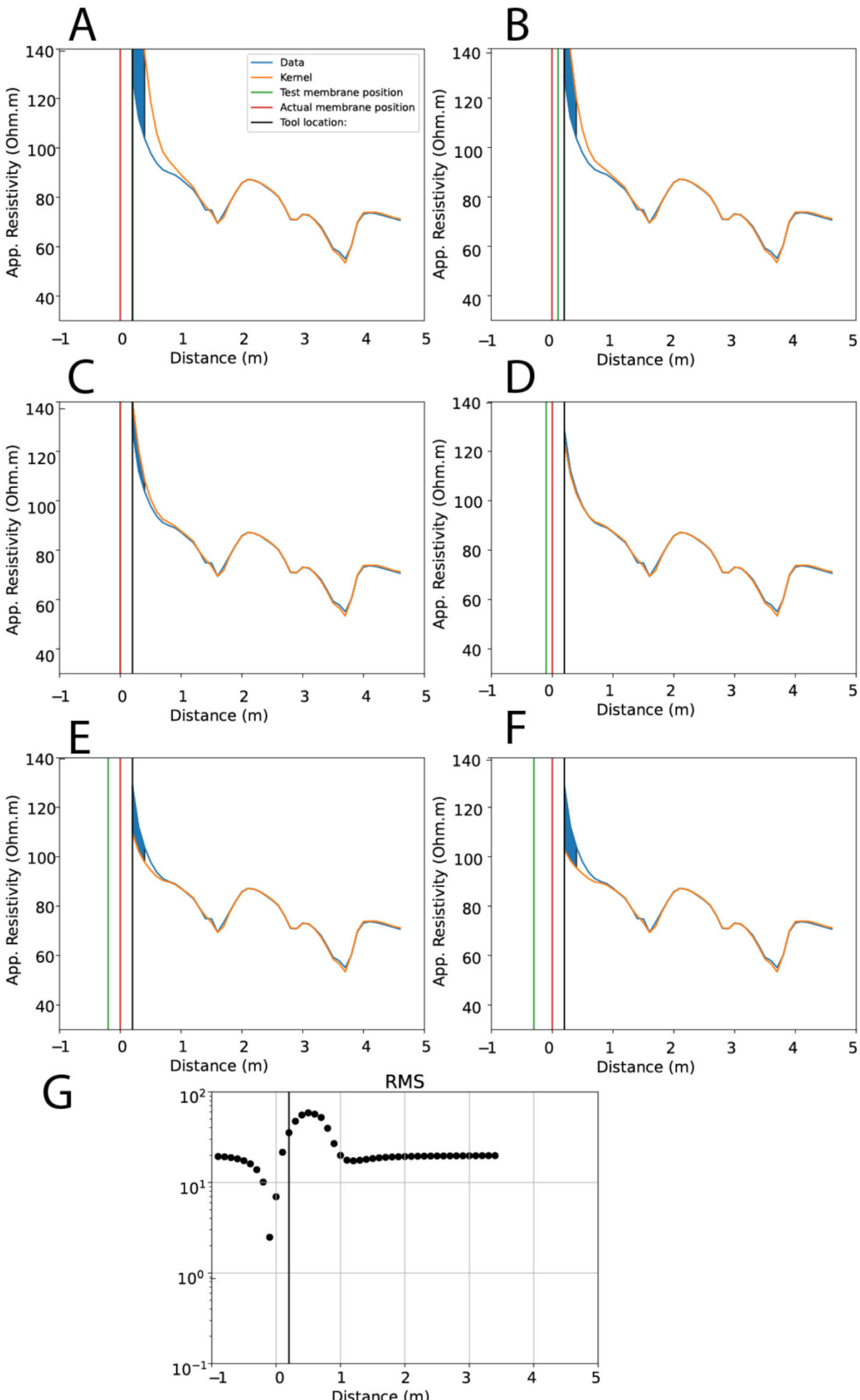

Figure 10. Same as Figure 8 with the tool (black vertical line) at $0.2 \mathrm{~m}$ from the membrane (red vertical line). (A-F) reflect tests for various simulated positions of the membrane. (G) shows the misfit function with a clear global minimum at $\mathrm{x}=-0.1 \mathrm{~m}$.

The background resistivity and kernels are calculated "on-the-fly" using the measurement data that have been acquired till the current tool position. The on-the fly workflow has 3 steps: 
A. First step: For each next position of the tool, a new set of Mode 1 and Mode 2 array measurement data is acquired.

B. Second step: Next, a tomographic inversion is performed from the surface up to current position of the tool. Inversion software Res3DiNV is used with dimensions of the simulated tank (i.e., the boundary effects are treated in 3D). This results in an update of the background resistivity (Figure 7, from top to bottom). For simplicity we only show one $\mathrm{XY}$ plane in the centre of the tank.

Mode 1 arrays are not influenced by the membrane, unless the tool is very close. Mode 1 is sensitive to the background resistivity close to the tool and therefore is most useful to obtain the background resistivity model up to the position of the tool. Mode 2 data, on the other hand, have larger looking ahead capabilities and these data are used calculate the updated kernels.

Figure 7 shows the update of the background resistivities for three positions of the tool. When the tool has just been inserted in the soil, there is only a small set of measurements available (Figure 7, top). This small data set is used to perform an inversion for a relatively small volume. When the tool is moved to a closer position (Figure 7, middle), more measurements are available and inversion of the background resistivity of a larger volume is determined. Even closer (Figure 7, bottom), the background resistivity image is nearly complete. The low resistivity zones of the clay slabs and cubes can be discerned. Also visible in the background resistivity image are the two plastic rails that guide the tool (because of the weight) that show up as two high resistivity lines.

C. Third step: At some point in moving the tool closer to the membrane, it might come too close which might result in puncturing the membrane. Therefore, after each measurement and inversion to obtain the background resistivity, a check is performed whether it is safe to push the tool to the next position. This is done by comparing the updated kernels to the measured data up to the latest position of the tool. After updating the kernels, an attempt is made to determine the position of the membrane relative to the tool. This is illustrated in Figures 8-10, for a decreasing distance from 1.2 to 0.6 to $0.2 \mathrm{~m}$ respectively. In theory, the position of the membrane corresponds to the kernel with the minimum misfit between the measured data and the kernel. However, if the tool is still far away from the membrane, too far even for Mode 2 to detect it, there will be no minimum in the RMS. This is the case in Figure 8, which shows the process of the fitting of the kernels. The true position of the membrane is indicated by the red vertical line, the current position of the tool by the black vertical line. The position of the simulated membrane (green vertical line) is moving away from the tool in panels A through F. The kernel corresponding to the simulated membrane position is shown in orange. These kernels were calculated using the background resistivity information from the start of the measurements until the current tool position at $1.2 \mathrm{~m}$. For example, Figure $8 \mathrm{~A}$ shows the line in orange that would have been measured if the membrane were $0.1 \mathrm{~m}$ ahead of the tool, at position $1.1 \mathrm{~m}$. For each simulated position of the membrane, the difference between the kernel (orange) and the data (blue) is calculated. This difference is visualised by the blue area in the plots. The misfit is expressed as the RMS error. For a distance of $0.1 \mathrm{~m}$ between the tool and the simulated membrane, (Figure $8 \mathrm{~A}$ ) the misfit is large, showing as a large blue area between the orange and the blue curve. When the simulated membrane is moved away from the tool in panels B to F, the misfit decreases, as is indicated by the smaller blue areas and the dots at various positions in Figure 8G. However, there is no clear minimum in the misfit. In this case, there are not enough data to "sense" the curvature of the kernel that is caused by the presence of the membrane. It is therefore safe to move the tool closer to the membrane and repeat the measurements and processing steps. This example also shows why it is necessary to update the kernels after each new measurement: the bumps in the curve at the right side of the tool are caused by the clay slabs and show the effect of varying background resistivity. The updated kernel closely follows the measured data. 
Figure 9 shows the same test for a tool position closer to the membrane. In this case the distance between them is $0.6 \mathrm{~m}$. Starting with the simulated membrane close to the tool (Figure 9A), the misfit is large. Moving the simulated membrane away from the tool, the misfit first decreases (Figure 9B,C), and increases again in Figure 9D-F. This pattern is reflected in the misfit plot in Figure 9G. When the simulated membrane is approximately on the actual location, a global minimum in the misfit is observed. In this case, the minimum occurs when the simulated membrane is at $-0.1 \mathrm{~m}$ and not at $0.0 \mathrm{~m}$ and reflects the uncertainty in the determined position of the membrane. Analysis of all electrode configurations at this position of the tool (not shown) indicates that, on average, the determined position can be $0.1 \mathrm{~m}$ off.

If better accuracy is required, the tool can be moved even closer to the membrane. This is shown in Figure 10, where the tool is only $0.2 \mathrm{~m}$ away from the membrane. Figure 10A shows the kernel when simulated membrane is exactly on the tool location and increasing the distance with $10 \mathrm{~cm}$ steps from Figure 10B-F. In The simulated membrane is exactly at the true membrane location in Figure 10C. A decrease in misfit is observed from Figure $10 \mathrm{~A}, \mathrm{~B}$, the global minimum at Figure $10 \mathrm{C}$ and an increase from Figure 10C-F. This is reflected by the plot in Figure 10G, which shows the same pattern as Figure 9G, but with a much sharper minimum indicating that the solution is more accurate.

\section{Determining the Position of the Membrane}

In the previous section, the on-the-fly calculation of the kernel and the misfit using simulated membrane positions was described. The misfit plots in those examples were made by using a single Mode 2 array with a $80 \mathrm{~cm}$ size of the tool. As mentioned, the distance between the electrodes (i.e., the tool size) has a positive effect in the looking ahead distance. In this section, we investigate this looking ahead capability with respect to different sizes of the tool (or different distances between the electrodes. Figure 11 presents the looking ahead capabilities of detecting the membrane for tool sizes varying from $10 \mathrm{~cm}$ to $80 \mathrm{~cm}$. The $x$-axis represents the various locations of the simulated membrane and the misfit is colour-coded. The bottom row of the plot $(80 \mathrm{~cm}$ size) is a summary of Figures 8-10. The other rows correspond to different electrode spacings in the Mode 2 array. If there is a global minimum in the misfit for a given configuration, then the corresponding shows a single pixel colour-coded as black and all other pixels within the row with various colours. If the minimum in the misfit is broad, i.e., there are several black pixels within the same row, then the position of the membrane is not defined very well and no unique solution could be obtained. In this case, it is an indication that is the distance between the membrane and the tool is too large to determine the membrane's position.

Each panel in Figure 11 shows the results for a specified distance between the tool and the membrane when the tool is approaching it. For larger distances $(1.3 \mathrm{~m}$ to $0.9 \mathrm{~m})$ most of the arrays cannot detect the position of the membrane, as indicated by the wide band of black pixels. However, is it still clear that the membrane is not present within the first several decimetres ahead of the tool. For $0.9 \mathrm{~m}$ to $0.7 \mathrm{~m}$, the largest tools start detecting the membrane accurately. The black misfit becomes narrower and is presented as a single pixel. At $0.7 \mathrm{~m}$ distance, the tool with sizes between 60 and $80 \mathrm{~cm}$ provide a good estimate of the membrane position. At closer distances, the smaller tools sizes also pinpoint the membrane at the right location. The blue spike indicates the most probable location, when all measurements are combined. Similar results were found also in the homogeneous cases, with the tank filled with either water or sand (not shown). Overall we conclude that

- a tool with a size of $45 \mathrm{~cm}$ was able to detect the membrane 10 to $20 \mathrm{~cm}$ from the true position at a distance of $0.6 \mathrm{~m}$.

- a tool of size $0.65 \mathrm{~m}$ and larger could do the same from a distance of $0.8 \mathrm{~m}$.

- closer to the membrane its location could be pinpointed at the true position or at $0.1 \mathrm{~m}$. 

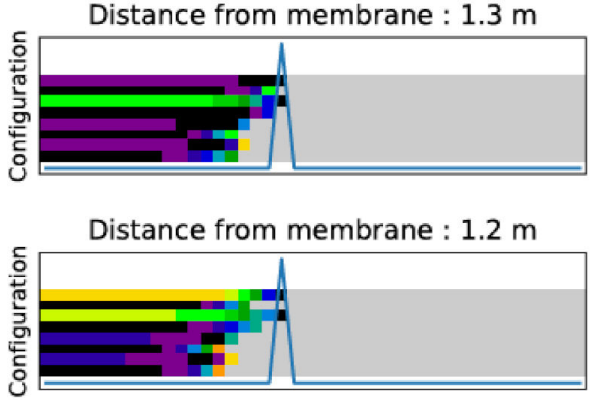

Distance from membrane : $1.1 \mathrm{~m}$
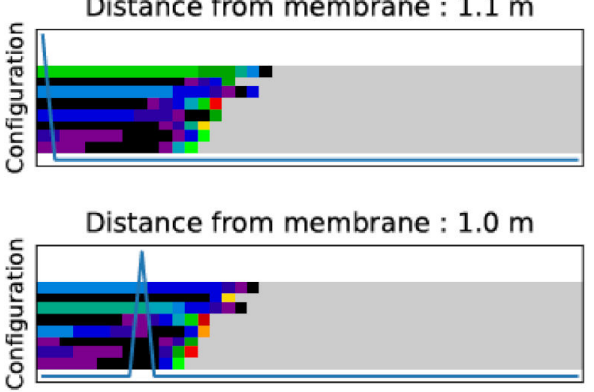

Distance from membrane : $0.9 \mathrm{~m}$
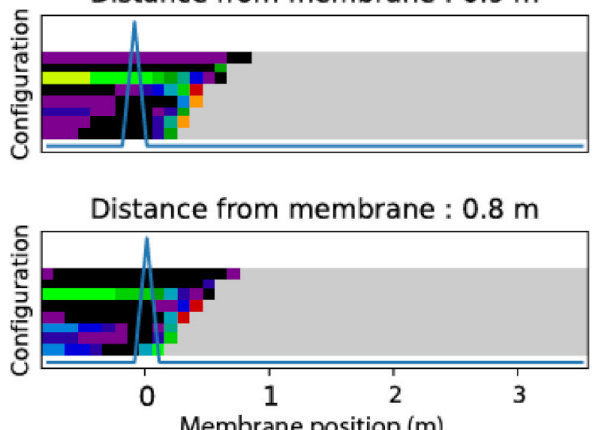

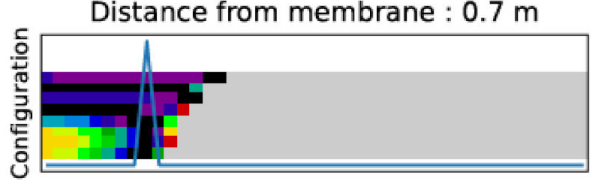

Distance from membrane : $0.6 \mathrm{~m}$

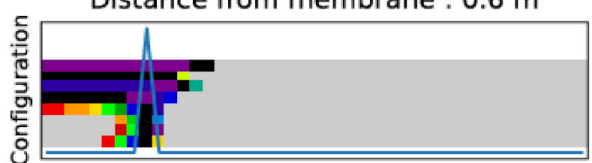

Distance from membrane : $0.5 \mathrm{~m}$

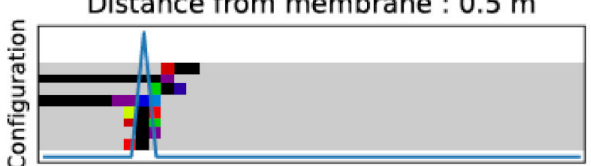

Distance from membrane : $0.4 \mathrm{~m}$

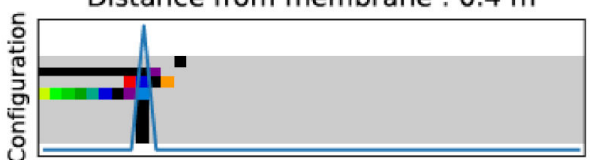

Distance from membrane $: 0.3 \mathrm{~m}$

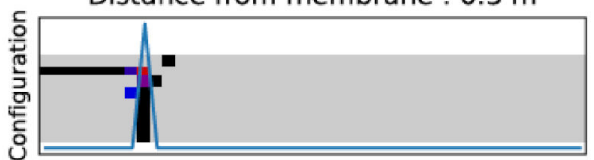

Distance from membrane : $0.2 \mathrm{~m}$

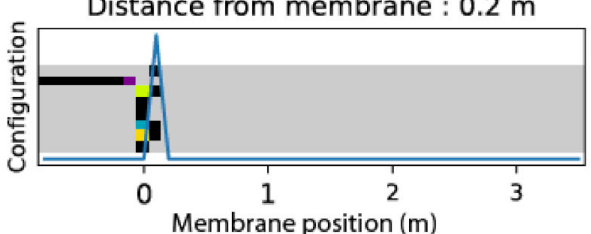

Membrane position (m)

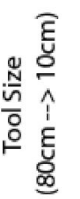

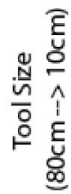

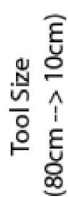

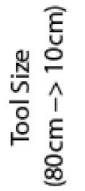

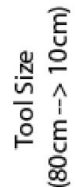

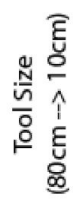

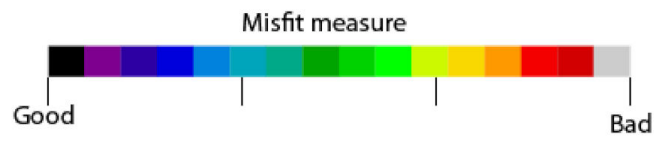

Figure 11. Visualisation of the misfit for Mode 2 arrays of varying electrode spacing (y-axis), with one distance between the tool and the membrane per panel. The misfit values are colour-coded with black indicating the minimum values. The true location of the membrane is at $0.0 \mathrm{~m}$. The peak shows the global minimum of the combined arrays with different sizes.

\section{Discussion}

Due to the finite dimensions of the tank, simulation of two remote electrodes showed reduced far looking ahead capability compared to a half-space and one remote electrode. As a result, the curvature of the kernel is far less pronounced than in the field situation. Still, the effect was sufficient in the laboratory to be able to pinpoint the position of the membrane. We expect that the membrane in the field will be detected from a larger distance than in the laboratory, or from the same distance but with a smaller tool. The smaller size for a field tool will make it easier to push into the ground.

Two types of configurations are required for the detection of the position of the membrane. The "far-looking" detection is sensitive to the presence of the membrane from a relatively large distance. However, the measurement is also influenced by local resistivity variations. The "near-looking" detection provides information about this background resistivity, so small changes to the curvature in the curve can't be mistaken for the membrane. The background resistivity information is needed for the kernel in the inversion procedure. Curvatures in the kernel can either be due to heterogeneity in the soil or the presence of the 
membrane. In order to isolate the effect of the membrane, the background must be included in the kernel. There is also a minimum distance needed between the current electrode and the voltage dipole in the "far" detection configuration. The best results are obtained for a tool with a width of at least $45 \mathrm{~cm}$. As mentioned though, we expect that the tool can have smaller dimensions, if we can utilize remote electrodes.

Field situations often have some degree of heterogeneity in the subsurface. It is recommended to perform some surface investigations prior to the membrane detection using pushing the tool in the ground. This surface ERT survey will also give general information about the background resistivity.

Other applications of this method are related to detection of very resistive or very conductive targets, where the exact burial depth is crucial. After adjusting the measuring strategy and processing based on the flowchart of Figure 5, targets smaller in size can also be detected.

\section{Conclusions}

In this paper, we have presented numerical and laboratory tests to investigate the looking ahead capabilities of ERT of a prototype tool in order to detect buried membranes. Two configurations have different looking ahead capabilities. Mode 1 with all electrodes on the tool is sensitive to the resistivity close to the tool and can be used to build-up the background resistivity image while measuring and pushing the tool into the ground. Mode 2, with electrodes on the tool and one or two remote electrodes on the surface, has large looking ahead capabilities and can sense the membrane from a larger distance.

The water-filled tank showed a successful proof of concept: the laboratory data closely followed the theoretical kernel. The two-step approach, with the updates of the background resistivity on the fly, also proved successful. The position of the membrane could be pinpointed in the sand\&clay tank with an accuracy of 10 to $20 \mathrm{~cm}$, depending on the dimension of the tool and the distance from the membrane.

The next step would be the development of a field tool. The design of the tool, the size, how to push it into the ground will have to be tailored made. The flowchart presented in Figure 5 can act as a methodology how to perform this task. This flowchart can also be used for other buried targets with a large resistivity contrast.

Author Contributions: Conceptualization, M.K., P.P.K., V.H. and B.B.; methodology, M.K., P.P.K. and V.H.; software, M.K.; validation, M.K. and P.P.K.; formal analysis, M.K., P.P.K. and V.H.; investigation, M.K. and P.P.K.; resources, B.B.; data curation, M.K. and P.P.K.; writing-original draft preparation, M.K. and P.P.K.; writing-review and editing, M.K. and P.P.K.; visualization, M.K.; supervision, P.P.K.; project administration, P.P.K.; funding acquisition, P.P.K. and B.B. All authors have read and agreed to the published version of the manuscript.

Funding: This research was funded by Rijkswaterstaat as part of Specialist Advice (no grant number available).

Data Availability Statement: Data available on request from the authors.

Acknowledgments: The idea of the method has been discussed in our team of geophysicists whichapart from the authors-includes Marco de Kleine, Chris Mesdag and Rik Noorlandt. The prototype of the tool has been designed by Mike van der Werf. He also assembled the tool and was assisted by Edvard Aldrich, Maarten Malingré and Miel Kuhr. The help of technicians Marcel Busink and Marcel Grootenboer in the laboratory was indispensable. We explained to them what we wanted to do and they made it happen. They installed the tank, the rails, the tool, the crane, the pulleys, the drainage system etcetera and operated this contraption during the ERT measurements. We also value the comments of two anonymous reviewers.

Conflicts of Interest: The authors declare no conflict of interest. 


\section{References}

1. Revil, A.; Karaoulis, M.; Johnson, T.; Kemna, A. Review: Some low-frequency electrical methods for subsurface characterization and monitoring in hydrogeology. Hydrogeol. J. 2012, 20, 617-658. [CrossRef]

2. Ward, S.H. Resistivity and Induced Polarization Methods. In Geotechnical and Environmental Geophysics: Volume I, Review and Tutorial; Society of Exploration Geophysists: Houston, TX, USA, 1990. [CrossRef]

3. Jardani, A.; Revil, A.; Santos, F.; Fauchard, C.; Dupont, J. Detection of preferential infiltration pathways in sinkholes using joint inversion of self-potential and EM-34 conductivity data. Geophys. Prospect. 2007, 55, 749-760. [CrossRef]

4. Smith, D.L. Application of the pole-dipole resistivity technique to the detection of solution cavities beneath highways. Geophysics 1986, 51, 833-837. [CrossRef]

5. Lapenna, V.; Lorenzo, P.; Perrone, A.; Piscitelli, S.; Rizzo, E.; Sdao, F. 2D electrical resistivity imaging of some complex landslides in Lucanian Apennine chain, southern Italy. Geophysics 2005, 70, B11-B18. [CrossRef]

6. Castilho, G.; Maia, D. A successful mixed land underwater 3D resistivity survey in an extremely challenging environment in Amazônia. In Proceedings of the 21th EEGS Symposium on the Application of Geophysics to Engineering and Environmental Problems, Philadelphia, PA, USA, 6-10 April 2008; pp. 1150-1158.

7. Bing, Z.; Greenhalgh, S. Cross-hole resistivity tomography using different electrode configurations. Geophys. Prospect. 2000, 48, 887-912. [CrossRef]

8. Vinegar, H.J.; Waxman, M.H. Method and Apparatus for Determining Shaliness and Oil Saturations in Earth Formations Using Induced Polarization in the Frequency Domain. U.S. Patent No. 4,359,687, 16 November 1982.

9. Vinegar, H.J.; Waxman, M.H. Induced polarization of shaly sands. Geophysics 1984, 49, 1267-1287. [CrossRef]

10. Anderson, B.I. Modeling and Inversion Methods for the Interpretation of Resistivity Logging Tool Response; IOS Press: Amsterdam, The Netherlands, 2001.

11. Schaeffer, K.P. An Experimental and Computational Investigation of Electrical Resistivity Imaging for Prediction Ahead of Tunnel Boring Machines. Ph.D. Thesis, Colorado School of Mines, Golden, CO, USA, 2016. Available online: https://ui.adsabs.harvard. edu/abs/2016PhDT........98S (accessed on 7 January 2022).

12. Kaus, A.; Boening, W. BEAM-Geoelectrical Ahead Monitoring for TBM-Drives. Géoméch. Tunn. 2008, 1, 442-449. [CrossRef]

13. Kopp, T. Real-Time Monitoring of Geological Conditions during Mechanized Tunnelling by Means of BEAM4 Methods. In Proceedings of the 1st Eastern European Tunnelling Conference, Budapest, Hungary, 18-21 September 2012.

14. Heiter, T.; Manila, H. Computing Discrete Fréchet Distance. In Christian Doppler Laboratory for Expert Systems; Technical Report CD-TR 94/64; Technical University Vienna: Vienna, Austria, 1994. 